Vol. 3, No. 1 | January - June 2019

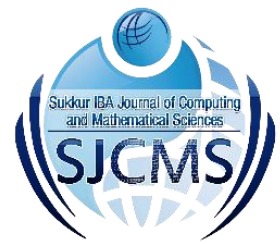

\title{
Analysis of Optimum Velocity and Pressure of the Air Flow through the Screens with the Help of Resistance Coefficient
}

Abid Ali Memon' ${ }^{1}$

\begin{abstract}
:
The present study based on applying the finite element's methodology to analyze the fluid flow through the two-dimensional channel rectangular shaped and escorted by the three blocks imposing with screen boundary conditions on them. These blocks or screens are affixed at unit distance from each other with two different angles $-45^{\circ}$ and $45^{\circ}$. The two-dimensional incompressible Navier Stokes equations are discretizing by finite element-based software COMSOL MultiPhysics 5.4 which embraced the Galerkin's least square operation. The air is working fluid which endures to enter from the exit of the channel with the average velocities of $1 \mathrm{~m} / \mathrm{sec}$ to 10 $\mathrm{m} / \mathrm{sec}$, the resistance and refraction coefficients are tested from 2 to 3 and 0.2 to 1 respectively. The objective of the study to analyze the maximum velocity and the maximum pressure when the fluid enters the region which comprises three screens inclined at angles by enhancing the resistance coefficient by fixing refraction coefficient. We uncovered that the maximum velocity magnitude, as well as pressure, is increasing with the enhancement of the resistance coefficient for a fixed refraction coefficient and the influences of average inlet velocities are to be determined with the help of resistance coefficient along with the refraction coefficient. Also, utilizing the statistical analysis method, we found that with the enhancement of the average inlet velocity by fixing refraction coefficient the relationship between maximum velocity magnitudes and resistance coefficient weakens, whereas the maximum pressure showed a strong connection with the resistance coefficient.
\end{abstract}

Keywords: resistance, refraction, angle, metalic screens

\section{Introduction}

In the field of design and engineering study, while any type of fluid be willed into any region it would be crucial to manage the velocity field and pressure distribution in the domain. For the reason some times, it is a need to study the fluid flow through porous medium or block with screen boundary conditions. The study of blocks with screen boundary condition is more reliable and relaxed as compared to that of the porous medium. Controlling a rate of flow of any fluid comes into any region. The metallic screens are used from decades. While learning the fluid flow actions via the screens it is demanded or essential to describe the association between the maximum velocity magnitude and maximum dynamical pressure with resistance coefficient $\kappa$

There are number of scientists, engineers and fluid flow analysts who accord to understand the dynamics of the fluid in the existence of screens. Elder [1] searched out the asymptomatic solution which discloses the relationship between the stream-wise velocity at the outlet of the channel related the small angle which is determined from the

${ }^{1}$ Department of Mathematics, Sukkur IBA University Sukkur, Pakistan

Corresponding Email: abid.ali@iba-suk.edu.pk 
focus of single screen. To upgrade airing system of the rooms or compartment, Teital [2] performed experiments in the lab while setting the inclined screens at angles from $45^{\circ}$ to $150^{\circ}$. The investigation was carrying out and validated the results with ANSYS CFX-11 working the porosities $0.4,0.52$ and 0.62. It was exposed that the mass flow rate while putting the screens at $45^{\circ}$ for all valued porosities is much better than that when screens are set at $135^{\circ}$. While analyzing fluids of nitrogen gas, air and water through Dutch Twilled Screens (DTS) and a Broad Mesh Geometry (BMG) a connection between non-dimensional Reynolds number Re and Euler number Eu was shaped by German and Fischer [3]. With the help of discrete element process, the fluid flow in the shape of rough particles past the screens at various angles was affected by Sawant et al [4]. He detected that the mass transfer is optimized by improving the arrangement of the angle. In short, his simulation links or provides basic to form a relationship between mass transfer and angle of the inclination of screens. While the fluid particles are passing or come into the junction through the screens, they actually give up energy. The head loss or energy loss of the particles presented with the screens are determined by the Santiago and Wang [5]. They found that the loss of energy can resist by transforming the screens from one angle to another angle. The whole research told us about the importance of saving energy. Abid, et al [6], studied the laminar and Newtonian fluid flow through the channel affixed with the three screens facing the resistance of 2.2 and refraction coefficient of 0.78 and all screens are movable from $-45^{\circ}$ to $45^{\circ}$ using the commercial software COMSOL Multiphysics 5.4. They contributed the empirical equations using the linear regression analysis for the maximum velocity, the maximum pressure in the whole domain and the drag force applying by each screen in terms of the angle of the screens. Brooke and Haughs [7] pioneered the new and very general approach for solving the compressible and incompressible fluid flow problems with one formulation recognized as Galerkin's Least Squares finite element method. According to the formulation if the velocity, pressure and temperature are the primitive variables then a unique discretized weak formulation, can be governed with the use of basic of finite element method with the help of least squares scheme.

The objective of the present study is to contribute about the distribution of optimized velocity as well as pressure while fluid passed through the three screens with the help of resistance coefficient along with the fixing refraction coefficient. The motivation behind the problem is that it provides the numerical visualization through the graphs for controlling the optimized

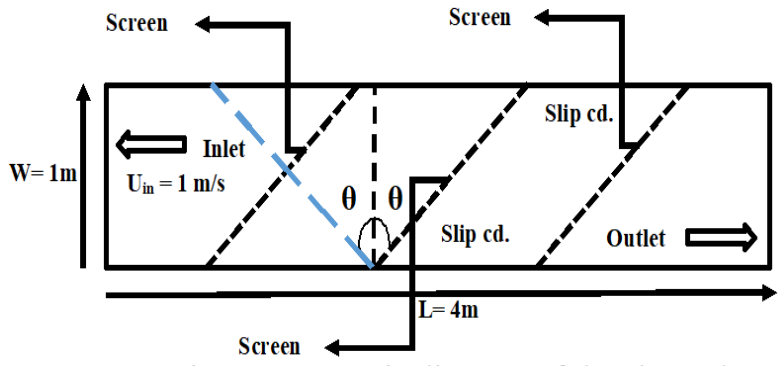

Fig. 1: Schematic diagram of the channel

velocity and pressure with the help of resistance coefficients for specific refraction coefficient. The problem is examined by using the screens boundary conditions with the special parameters of resistance coefficient (2 to 3 ) and refraction coefficient ( 0.2 to 1$)$. The results are achieved using the package of finite element methods COMSOL MultiPhysics 5.4 and presented through graphs and tables. The result is contrasted with the asymptotic solution provided by Elder [1959].

\section{Methodology}

\subsection{Geometry and Boundary Condition}

Reckoning the rectangular channel, we place the length and width of the geometry with $4 \mathrm{~m}$ and $1 \mathrm{~m}$ respectively. The three blocks with screen boundary conditions are set at the $1 \mathrm{~m}$ distance from each other which are moveable at $-45^{\circ}$ and $45^{\circ}$. The schematic diagram in the Fig. 1 is shown with inlet and outlet boundary conditions. Air as working fluid is approved to flow from the doorway of the channel with speed from 1 to 10 $\mathrm{m} / \mathrm{sec}$ in series and while at the exit of the channel pressure is considering zero pressure.

\subsection{Meshes of Geometry}

The designated rectangular domain of the geometry with three blocks has been split into irregular triangular elements about 2386 with minimum and maximum quality elements of 0.76 and 0.12 respectively. As this problem of the fluid flow are analyzed using rich techniques of Galerkin's Least Square of finite element approach. It was the fundamental necessity of any numerical strategy to put in the meshing process over the interested area. Also, it is the naked truth that to obtain more and more unambiguous or predictable numerical result the mesh of the geometry should be more and finer. Although it would get enough time to perform the simulation and to prepare the acquired convergence of the problem. However, the present problem has been put into analysis by doing a normal meshing in the domain, and we got the results which will be comparable to asymptotic solution sought by Elder [1959]. 


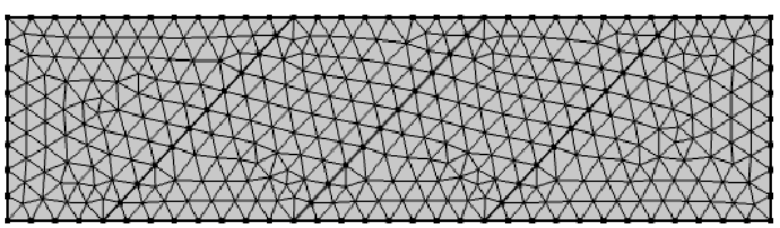

Fig. 2 Irregular Triangular Meshes of the Geometry

The selected geometry is meshed using the irregular triangular elements shown in Fig.2 and the mesh statistics are given in Table I.

Table I: Mesh statistics of the Geometry

\begin{tabular}{|c|c|}
\hline Property name & Value \\
\hline Minimum quality element & 0.7629 \\
\hline Maximum quality element & 0.9793 \\
\hline Number of triangular elements & 2382 \\
\hline Edge elements & 215 \\
\hline Vertex elements & 10 \\
\hline
\end{tabular}

\subsection{Governing Equations}

The steady state and two dimensional incompressible Navier Stokes equations are worked out numerically with constant viscosity $v$ and density $\rho$ employing the screen boundary conditions putting in on the three blocks of the channel. Expected to the non-linearity of the second order Navier Stokes equations, it is very stiff or almost unfeasible to get the accurate solution function for the velocity field and pressure without regulations which is not but a settlement. In the meadow of numerical methods, several numerical procedures were implemented from the periods. Every one of them was assessed, as a best numerical procedure to get the solution of the fluid dynamics problems of their particular times. In this article, we are attending to debate the numerical results acquired through least square procedure of the finite element methods. For the grounds we use COMSOL MultiPhysics 5.4 to descritize the second order Navier Stokes equations utilizing the screen boundary conditions. The governing partial differential to calculate the velocity and pressure is given below:

$$
\frac{\partial \vec{V}}{\partial t}+(\vec{V} \cdot \nabla) \vec{V}=-\frac{1}{\rho} \nabla p+n \nabla^{2} \vec{V}+F
$$

$$
\nabla \cdot \vec{V}=0
$$

$$
\rightarrow
$$

Where $\mathrm{V}$ is the velocity field with $\mathrm{u}$ and $\mathrm{v}$ as $\mathrm{x}$ and $\mathrm{y}$ components respectively. Due to the steady state study, we can assume the time derivative of the velocity field equal to zero. Hence, we have (3)

$$
\frac{\partial \vec{V}}{\partial t}=0
$$

Further, our requirement is that the viscous impact should not be seen in the upper and lower wall of the channel. Therefore, slip $\rightarrow$

boundary condition will facilitate us. If $n$ is the vector normal to the velocity field then:

$$
\begin{aligned}
& \vec{V} \cdot \vec{n}=0 \\
& \vec{K} \cdot(\vec{K} \cdot \vec{n}) \vec{n}=0
\end{aligned}
$$

Where

$$
\vec{K}=v(\nabla \vec{V}+(\nabla \vec{V}) T) \vec{n}
$$

Finally, we will describe the screen boundary condition referring equation (7), (8) and (9). In the fluid flow investigation screen boundary condition suppress the tangential component of the velocity field and helpful to boost the speed in the channel.

$$
\begin{aligned}
& {[\rho \vec{V} \cdot \vec{n}]_{-}^{+}=0} \\
& {\left[\begin{array}{l}
\overrightarrow{(n)}^{T} \\
\left.K \vec{n}+\rho(\vec{V} \times \vec{n})^{2}\right]_{-}^{+}
\end{array}\right.} \\
& =-\frac{k}{2} \rho_{-}(\vec{V}-\times \vec{n})^{2} \\
& \rightarrow \rightarrow \quad \rightarrow \rightarrow \\
& n \times V_{+}=\eta\left(n \times V_{-}\right)
\end{aligned}
$$

In equations + and - shows the presents of the parameter up and downstream respectively.

After doing the numerical calculation, we will apply the statistical technique to calculate the correlation coefficient $r$ by using the equation (10) as given below: 


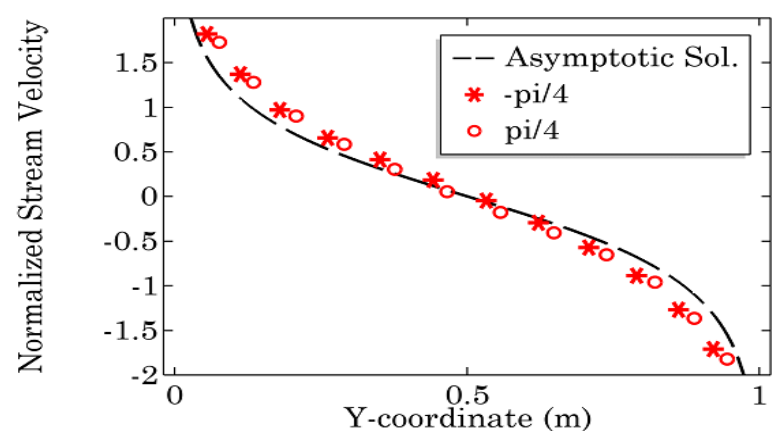

Fig 3. Comparison with asymptotic solution

$$
r=\frac{\sum_{i}\left(x_{i}-\bar{x}\right)\left(y_{i}-\bar{y}\right)}{\sqrt{\sum_{i}\left(x_{i}-\bar{x}\right)^{2}} \sqrt{\sum_{i}\left(y_{i}-\bar{y}\right)^{2}}}
$$

Where

$$
\bar{x}=\frac{\sum_{i} x_{i}}{n} \text { and } \bar{y}=\frac{\sum_{i} y_{i}}{n}
$$

To calculate the $V_{\text {corr }}$ for $45^{0}$ and $-45^{0}$ we will take $x=V_{\text {max }}$ and $y=\kappa$. Also, for calculating $P_{\text {corr }}$ either at $45^{\circ}$ and at $-45^{0}$ we will consider $x=P_{\max }$ and $y=k$.

\section{Results and Discussion:}

Initially, we attempt to validate our method of solution by numerically calculating the stream -wise velocity at outlet of the channel and compared with that of asymptotic solution given by [1], see Fig. 3. The result shows the strong agreement with the numerical procedure.

\subsection{Velocity and Pressure Distribution at $\mathbf{4 5}^{\circ}$}

In the first stage, all screens are standing at $45^{\circ}$. The fluid flow is investigated through the channel with three blocks facing screen boundary condition. Maximum velocity flowing through the whole interested domain is calculated by keeping constant one of the refraction coefficients from 0.2 to 1 for all of the resistance coefficient $\kappa$ from 2 to 3. Fig. 4 shows the maximum velocity magnitude in the domain calculated for the initial speed at uin at $1 \mathrm{~m} / \mathrm{sec}$ at all resistance coefficients $\kappa$ from 2 to 3 at particular refraction coefficient $\eta$. The figure conveys the message with fixing $\eta$ the maximum velocity magnitude is increasing with increasing in resistance coefficient $\kappa$ due to the increment in the hydraulic energy of the fluid. As soon as we increase refraction coefficient $\eta$, the maximum velocity magnitudes is decreasing due to the change of the direction of the air while crossing the screen. In Fig. 5 responses of average inlet speeds 4,8 and 10 is given which conveys the message that for a particular refraction coefficient the response of maximum velocity is decreasing for all inlet velocities and for all corresponding resistance coefficient.

It might be a tradition of the research study if there is argument for the velocity distribution in the domain there should be argument for pressure distribution. In the article, we have also produced the graphs for maximum pressure resulting in the domain beside resistance coefficient for particular refraction coefficient that is worked out from the numerical scheme. The Fig. 6 shows that for a Particular refraction coefficient $\eta$ the maximum pressure is increased linearly for all resistance coefficients due to the declining of the kinetic energy of the fluid when the fluid comes to strike with the screens. In the article we have also put on the graphs for maximum pressure occurring in the domain against resistance coefficient for particular refraction coefficient that are calculated from the numerical scheme. The responses of all uni, the average inlet speed at $4 \mathrm{~m} / \mathrm{sec}, 8 \mathrm{~m} / \mathrm{sec}$ and $10 \mathrm{~m} / \mathrm{sec}$ are calculated in the shape of maximum pressure shown through the Fig. 7. This also clearly concentrates on the forecast that with decreasing in refraction coefficient the maximum pressure is decreasing.

We also enhance our investigation in the direction of the statistics judgment by finding the correlation coefficients between $\kappa$ with maximum velocity and pressure at particular $\eta$ ,inlet velocity at the angle look at Table-2. It is very understandable from the results due to boost in inlet velocity the correlation between resistance coefficient $\kappa$ and maximum velocity is turning down, while the maximum pressure shows the strong relationship with $\kappa$. Furthermore, the given Table-2 also admitted about the average maximum velocity right through as well as pressure in the channel. However, we were unable to define the correlation coefficient between $\kappa$, and max velocity at $\eta$ at $1 \mathrm{~m} / \mathrm{sec}$.

\subsection{Velocity and Pressure Distribution at $\mathbf{- 4 5 ^ { \circ }}$}

In the 2nd phase, we also attend to look at the maximum velocity causing in the domain with the aid of increasing resistance $\kappa$ coefficient with fixing of refraction coefficient $\eta$ for all the screens at $-45^{0}$. Fig. 8 shows the consequences of Vmax when the inlet velocity uin $=1 \mathrm{~m} / \mathrm{sec}$. Of course, likely to the screens is prepared at $45^{\circ}$, in this case the maximum velocity magnitude is also raised by fixing refraction coefficient as a 

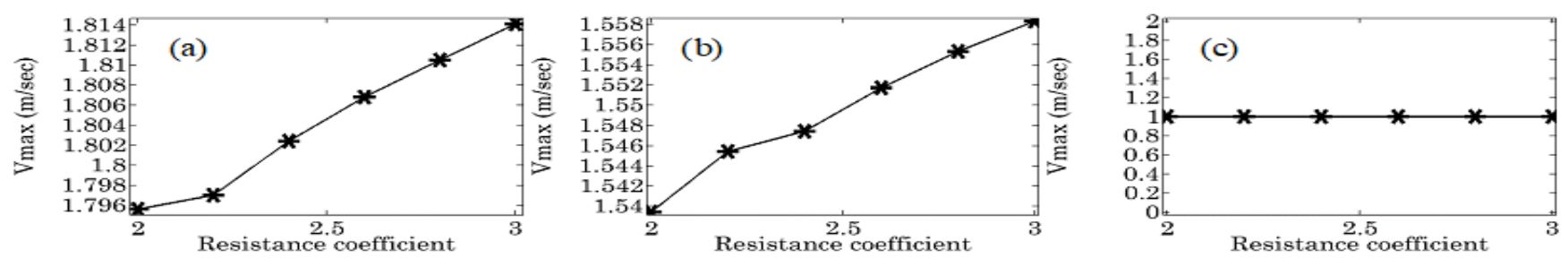

Fig. 4 Optimum velocity magnitude against the resistance coefficient at the unit initial speed for a particular refraction coefficient at (a) 0.2 (b) 0.4 and (c) 1
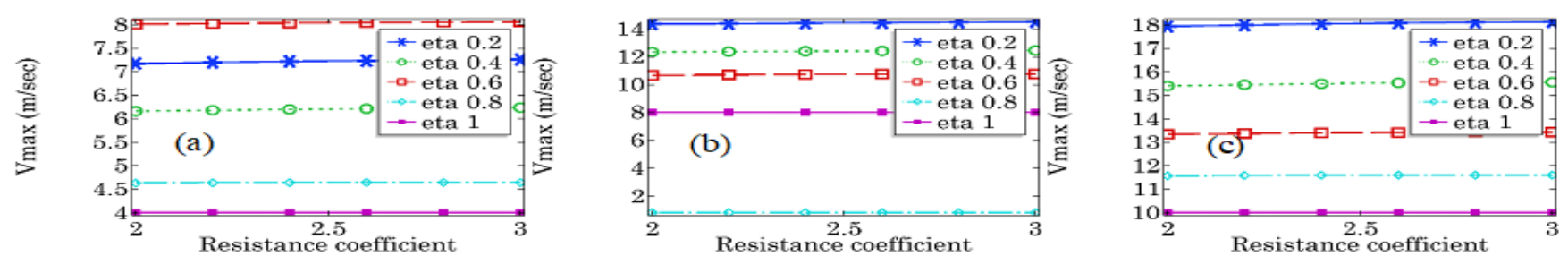

Fig. 5 Optimum velocity magnitude against the resistance coefficient for all refraction coefficients at initial velocities of (a) $4 \mathrm{~m} / \mathrm{sec}$ (b) $8 \mathrm{~m} / \mathrm{sec}$ and (c) $10 \mathrm{~m} / \mathrm{sec}$
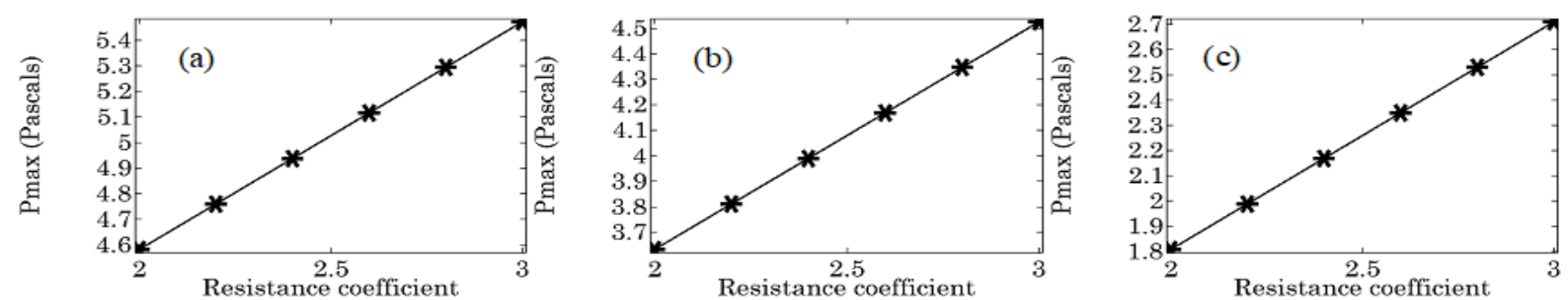

Fig. 6 Optimum Pressure $(\mathrm{Pa})$ against the resistance coefficient at the unit initial velocity for a particular refraction coefficient at (a) 0.2 (b) 0.4 and (c) 1
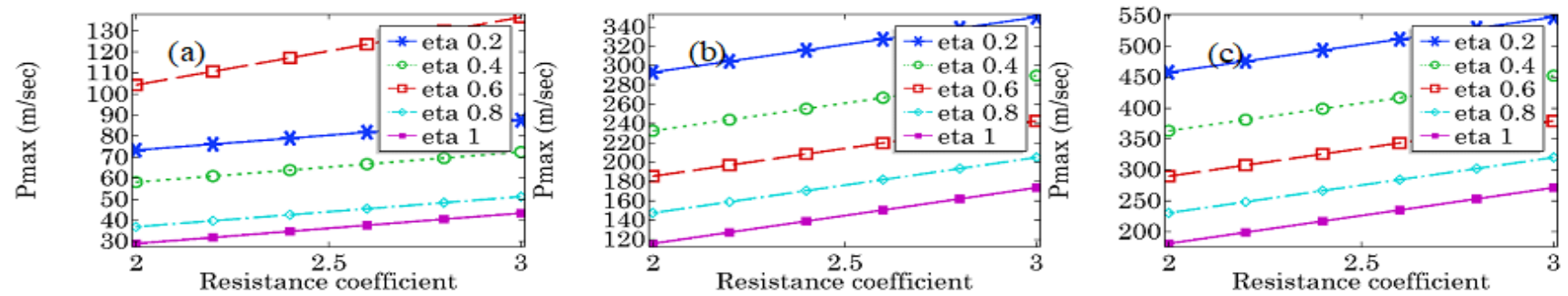

Fig. 7 Optimum Pressure $(\mathrm{Pa})$ against the resistance coefficient for all refraction coefficients at initial velocities of (a) $4 \mathrm{~m} / \mathrm{sec}$ (b) $8 \mathrm{~m} / \mathrm{sec}$ and (c) $10 \mathrm{~m} / \mathrm{sec}$ 

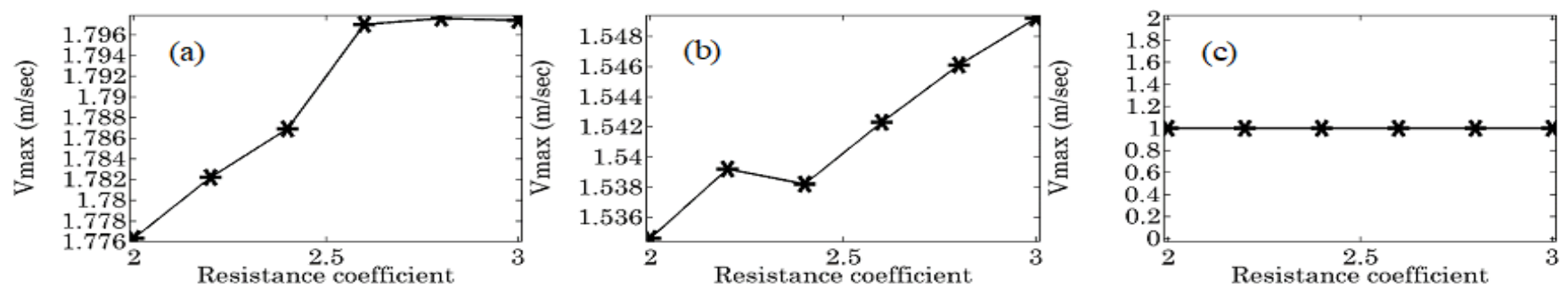

Fig. 8 Optimum velocity magnitude against the resistance coefficient at the unit initial speed for a particular refraction coefficient at (a) 0.2 (b) 0.4 and (c) 1
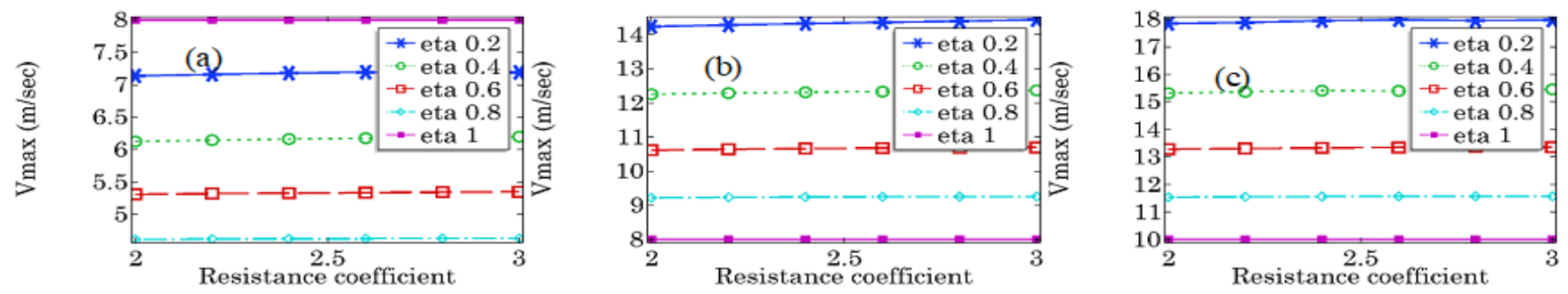

Fig. 9 Optimum velocity magnitude against the resistance coefficient for all refraction coefficients at initial velocities of (a) $4 \mathrm{~m} / \mathrm{sec}$ (b) $8 \mathrm{~m} / \mathrm{sec}$ and (c) $10 \mathrm{~m} / \mathrm{sec}$
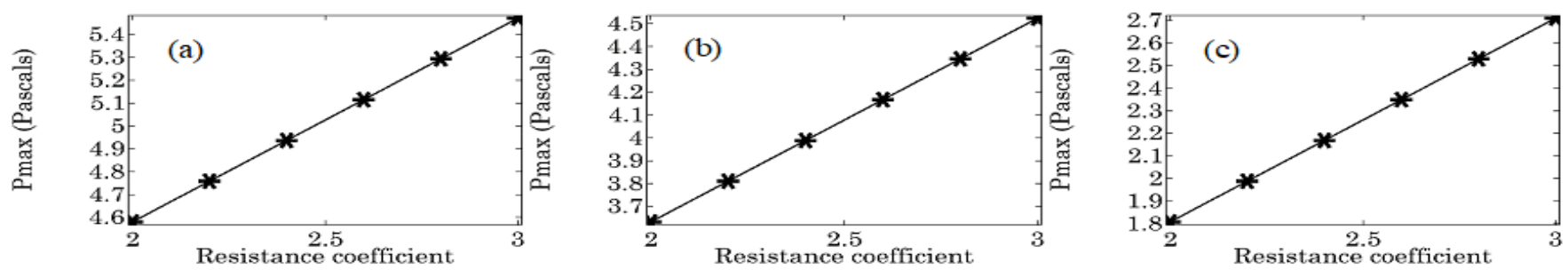

Fig. 10 Optimum Pressure $(\mathrm{Pa})$ against the resistance coefficient at the unit initial speed for a particular refraction coefficient at (a) 0.2 (b) 0.4 and (c) 1
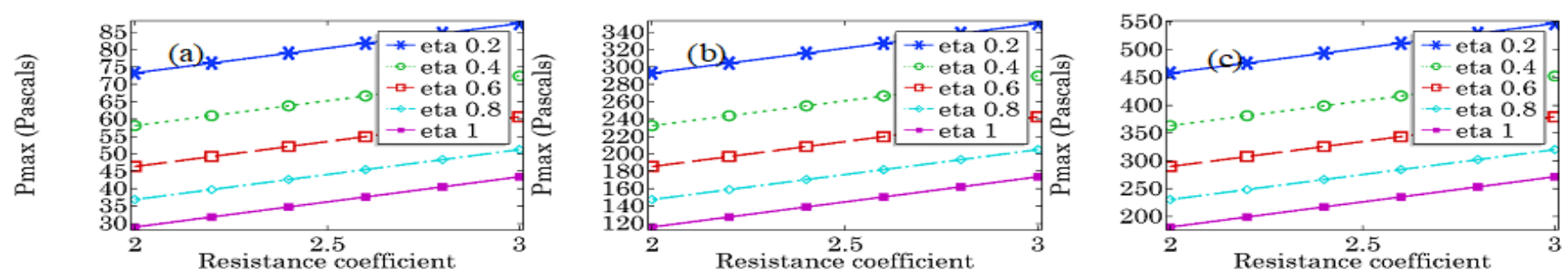

Fig. 11 Optimum Pressure $(\mathrm{Pa})$ against the resistance coefficient for all refraction coefficients at initial velocities of (a) $4 \mathrm{~m} / \mathrm{sec}$ (b) $8 \mathrm{~m} / \mathrm{sec}$ and (c) $10 \mathrm{~m} / \mathrm{sec}$ 
constant and altering the resistance coefficient from 2 to 3. Further, the responses of average inlet velocity magnitudes are also assessed at 48 and $10 \mathrm{~m} / \mathrm{sec}$ to get the maximum speed running in the domain. The maximum pressure endured on the channel is also determined by fixing eta and enabling kappa to boost from 2 to 3. Fig. 10 evidently renders that maximum pressure is boosting linearly with the resistance coefficient kappa. Thus, a linear relationship can be determined by least square procedure between $\kappa$ and optimized pressure for each $\eta$

.The optimized pressure possesses the same relationship for the inlet average velocities uin 4, 8 and $10 \mathrm{~m} / \mathrm{sec}$. See Fig.11. From the essence of thought of statistics reasoning, the Table-2 presents the correlation coefficient for the relationship between $\kappa$ and maximum velocity magnitude as well as pressure. The results demonstrate that for a specific $\eta$ the pressure habitually shows causal relationship with kappa whereas the correlation coefficient is dropping for max: velocity as we increase inlet velocity. However, we were powerless to define the correlation coefficient between $\kappa$ max velocities at eta at $1 \mathrm{~m} / \mathrm{sec}$.

In the present paper, we were attempting to demonstrate the air flow attitude through the rectangular channel fitted with three blocks by tricking boundary conditions. The two-dimensional incompressible Navier Stokes equations were numerical worked out, and the simulation was carried out with the help of commercial software COMSOL MultiPhysics 5.4. The density and viscosity of the fluid were gripped as constants. The contemplation was parametric, and the parameter was specified as resistance coefficient $\kappa$ from 2 to 3 with refraction coefficient from 0.2 to 1 . The behavior of the maximum velocity magnitude and pressure was monitored by fixing $\eta$ and moving $\kappa$ and further we estimate the correlation coefficient between resistance coefficient and maximum speed and pressure in the domain. At the moment we are in place to put forward the following terminal points:

1. In the channel while checking out the maximum velocity field resulting when screens are affixed at either $45^{\circ}$ or $-45^{0}$ is increased for increasing resistance coefficient at a particular refraction coefficient. But maximum velocity magnitude does not keep linear relationship with resistance coefficient.

2. For a particular average inlet velocity magnitude, the maximum velocity in the domain is decreased while increasing the resistance coefficient at a specific refraction coefficient.

3. For all inlet average speeds in setting the screens either in $45^{0}$ or $-45^{0}$ degree, the maximum pressure is increasing linearly by enhancing resistance coefficient at fixed refraction coefficient. At a particular average inlet speed, the optimum pressure is decreasing with increasing in refraction coefficient for all resistance coefficients.

4. With the helping hand of theory of statistics analysis, we have calculated the correlation coefficient between the maximum velocity as well as pressure with the resistance coefficient at fixed refraction coefficient. We conclude that with the increase in inlet average velocity magnitude, the relationship between the maximum speed in the domain and resistance coefficient weakens at fixing eta. But resistance coefficient possesses strong relation with maximum pressure by fixing eta.

\section{References}

[1] Elder, J. W. "Steady flow through non-uniform gauzes of arbitrary shape." Journal of Fluid Mechanics 5, no. 3 (1959): 355-368.

[2] Teitel, Meir. "Using computational fluid dynamics simulations to determine pressure drops on woven screens." Biosystems engineering 105, no. 2 (2010): 172-179.

[3] Fischer, Alexander, and Jens Gerstmann. "Flow resistance of metallic screens in liquid, gaseous and cryogenic flow." In 5th European Conference for Aero-Space Science, München, pp. 15. 2013.

[4] Gunaji Ashok Sawant, V Murali Mohan, and Sandip Ashok Sawant. "Study and analysis of deck inclination angle on efficiency of vibration screen." IJDER 4, no. 2 (2016): 104-106

[5] Santiago, Channing RC, Ted Chu, and K. Wang. "Study of the Head Loss Associated with a Fluid Flowing through a Porous Screen." no. August(2007): 1-26.

[6] Memon, Abid Ali, Hisamuddin Shaikh, and Asif Ali Memon. "Finite Element's Analysis of Fluid Flow through the Rectangular Channel with Inclined Screens settled at Angles." In 2019 2nd International Conference on Computing, Mathematics and Engineering Technologies (iCoMET), pp. 1-5. IEEE, 2019.

[7] Hauke, G., and T. J. R. Hughes. "A unified approach to compressible and incompressible flows." Computer Methods in Applied Mechanics and Engineering 113, no. 3-4 (1994): 389 
